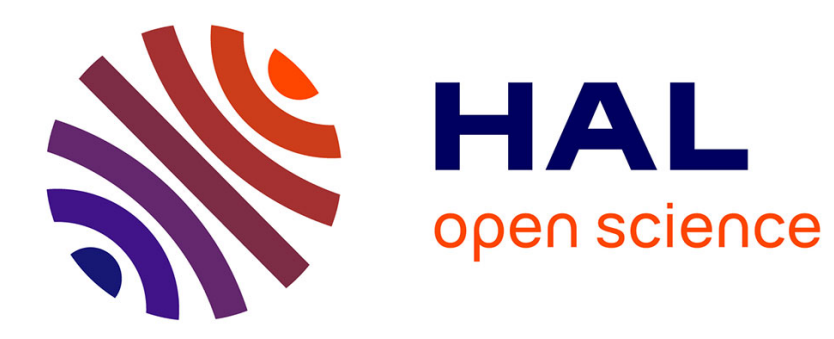

\title{
Spatial quantum noise of laser diodes
}

Jean-Philippe Poizat, Tiejun Chang, Olivier Ripoll, Philippe Grangier

\section{To cite this version:}

Jean-Philippe Poizat, Tiejun Chang, Olivier Ripoll, Philippe Grangier. Spatial quantum noise of laser diodes. Journal of the Optical Society of America B, 1998, 15 (6), pp.1757-1761. 10.1364/JOSAB.15.001757 . hal-00558810

\section{HAL Id: hal-00558810 \\ https://hal-iogs.archives-ouvertes.fr/hal-00558810}

Submitted on 3 Apr 2012

HAL is a multi-disciplinary open access archive for the deposit and dissemination of scientific research documents, whether they are published or not. The documents may come from teaching and research institutions in France or abroad, or from public or private research centers.
L'archive ouverte pluridisciplinaire HAL, est destinée au dépôt et à la diffusion de documents scientifiques de niveau recherche, publiés ou non, émanant des établissements d'enseignement et de recherche français ou étrangers, des laboratoires publics ou privés. 


\title{
Spatial quantum noise of laser diodes
}

\author{
Jean-Philippe Poizat,* Tiejun Chang, Olivier Ripoll, and Philippe Grangier \\ Institut d'Optique, B.P. 147, F91403 Orsay Cedex, France
}

Received December 3, 1997; revised manuscript received February 25, 1998

\begin{abstract}
We experimentally study the transverse distribution of intensity noise in the far field of a single-mode semiconductor laser. We show that a large amount of noise is present in the higher-order nonlasing transverse modes (parallel to the diode junction). Furthermore, correlations between the $\mathrm{TE}_{00}$ and the $\mathrm{TE}_{10}$ modes are observed. (C) 1998 Optical Society of America [S0740-3224(98)00106-4]

OCIS codes: $140.2020,270.3100,270.6570$.
\end{abstract}

Quiet driving of laser diodes has proved in the past decade to be an efficient and convenient way to produce light whose intensity noise is below the standard quantum limit (SQL) (see, for example, Refs. 1-3). The pumping mechanism of semiconductor lasers allows a faithful conversion of the electron statistics of the driving current to the photon statistics of the outgoing light., ${ }^{4,5}$ This simple picture does not, however, work for all laser diodes. For example, the importance of multimode effects ${ }^{6}$ on the noise behavior has been recently demonstrated for longitudinal $^{7-9}$ and polarization modes. ${ }^{10}$ In these experiments the different modes were lasing, and the main effect was an anticorrelation between them owing to mode competition. Let us also mention that anticorrelations have been observed between transverse modes with orthogonal polarizations in the recent demonstration of subPoissonnian operation of vertical-cavity surface-emitting lasers. $^{11}$

In this paper we investigate the influence of transverse modes on the intensity noise of stripe laser diodes. Understanding the dynamics of higher-order transverse modes is of great importance when optimizing the coupling of a laser diode into an optical fiber or when diode pumping a solid-state laser. ${ }^{12}$ Moreover, investigating noise properties of a laser is a very sensitive way to access information about its dynamics. The appearance of noise in a mode can often be interpreted as a precursor for mode-switching phenomena.

We perform two complementary experiments. The first experiment analyzes the intensity noise of a beam screened in the far field by a movable razor blade. The fitting of the noise profile allows us to identify precisely the noisy spatial modes and to infer the presence of correlations between the lasing mode and a subthreshold higher-order spatial mode. In the second experiment these correlations are measured directly with a twoquadrant photodiode to extract the noise information of the higher-order spatial mode. This method, however, lacks information about the details of the spatial profile because it cannot tell which are the higher-order modes contributing to the intensity noise, as in the first experiment.

The single-mode semiconductor laser used is a FabryPerot multiple-quantum-well index-guided AlGaAs device
(SDL 5411-G1) emitting at $810 \mathrm{~nm}$. It has a single lasing transverse mode. It is collimated by a high numerical aperture (N.A. $=0.65)$ aspherical lens and is stabilized by the $24 \%$ feedback of an external grating. In this configuration its intensity noise is $16 \%$ below the SQL and its longitudinal side-mode rejection rate is greater than 35 $\mathrm{dB}^{3,9}$ Note that the noise reduction given throughout this paper is corrected for the transmission of the optical components and the detectors quantum efficiency. It corresponds therefore to the real noise at the output of the grating. No correction is done for the grating efficiency (60\% in zeroth order).

In the first experiment we analyze the spatial distribution of noise within the transverse profile of a semiconductor laser beam by measuring its intensity noise after screening part of it with a movable razor blade. A simplified experimental setup is shown in Fig. 1. The razor blade is on a motorized translation stage. It can be scanned along the $x$ direction as in the figure, or along the $y$ axis. The intensity noise detection is a standard balanced scheme that allows a convenient comparison between the intensity noise and the SQL. ${ }^{13}$ The photodiodes are large-area high-efficiency $p$ - $i$ - $n$ devices (Silicon Sensor SSO-PD20-7). ${ }^{14}$ The DC current is filtered out, and the RF photocurrent is amplified and sent to an electronic spectrum analyzer. The RF noise-analysis frequency of the results presented below is $14 \mathrm{MHz}$, but the frequency dependence of the effects is essentially flat within the bandwidth of our detectors (2-20 MHz).

The results of a typical scan along the $x$ axis are displayed in Figs. 2 and 3, for a laser diode driving current of $I=100 \mathrm{~mA}$ (the threshold is $I_{\text {th }}=17 \mathrm{~mA}$ ). The SQL exhibits an error function (erf) shape since it is proportional to the intensity that has the Gaussian profile of the lasing $\mathrm{TE}_{00}$ mode. The intensity noise has a very different shape. This means that the noise transverse distribution is different from the mean intensity distribution and that noise is present in higher-order spatial modes. In order to identify these modes, and to quantify their noise and correlations, the experimental scans are fitted with a simple theoretical model.

In this model, only the main $\mathrm{TE}_{00}$ mode is lasing. The other transverse modes are below threshold and consist of amplified spontaneous emission. Their central frequen- 


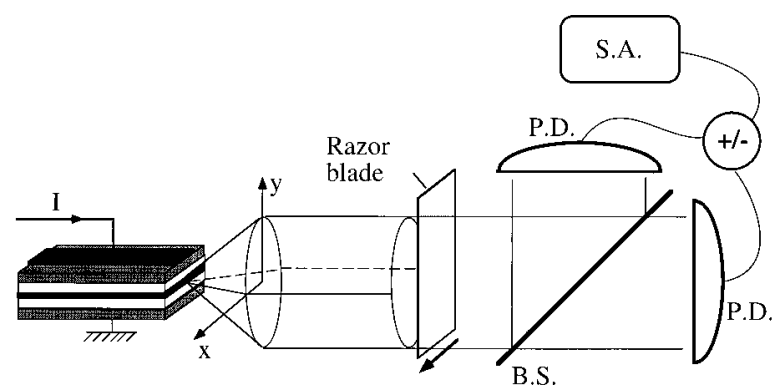

Fig. 1. Simplified experimental setup. The external grating is not shown, for the sake of clarity. B.S., beam splitter; P.D., photodiode; S.A., spectrum analyzer.

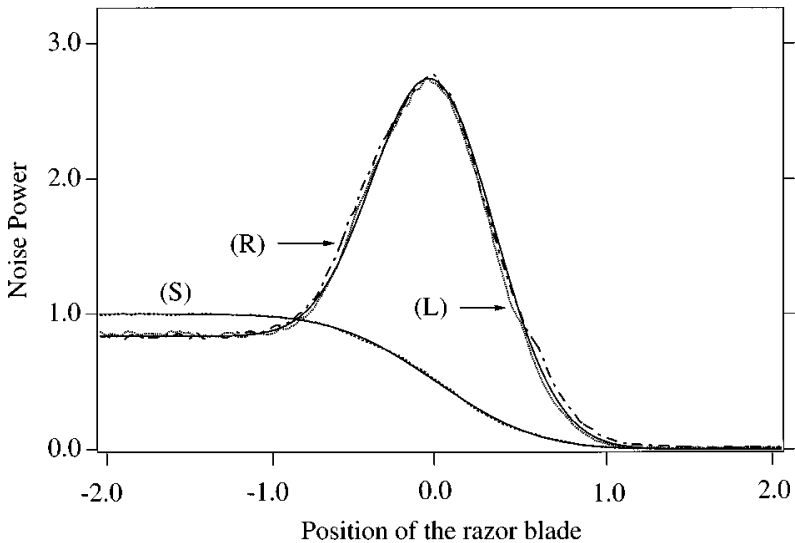

Fig. 2. Razor-blade scan along $x$ (with $w=1$ ). Trace (S) is the $\mathrm{SQL}$ (proportional to the intensity). Trace (L) [or (R)] corresponds to the razor blade entering the beam from the left (right). Trace $(\mathrm{R})$ is flipped over in order to be compared conveniently with trace (L). The two traces are almost superimposed, which is the signature for a vanishing correlation. For each trace the thin solid curve is the best fit. The fitting parameters are $v_{0}$ $=-0.16 \pm 0.02, v_{1}=14.3 \pm 0.2, v_{2}=4 \pm 1$, and $C=0.00$ \pm 0.02 .

cies are different from the main mode's one, but their spectrum is very broad (of the order of the free spectral range of the cavity), because the output coupling mirror has a very small reflectivity $(R=4 \%)$. There is therefore a frequency overlap between the higher-order transverse modes and the $\mathrm{TE}_{00}$ mode, which acts as a local oscillator that homodynes the noise of the higher-order modes. ${ }^{15}$ If the only nonlasing mode is the $\mathrm{TE}_{10}$ mode, the intensity noise when the razor blade is at position $X$ is given by (see Appendix A)

$$
\begin{aligned}
V(X)= & E_{0}^{2}\left\{\int_{X}^{\infty} u_{0}^{2}(x) \mathrm{d} x+v_{0}\left[\int_{X}^{\infty} u_{0}^{2}(x) \mathrm{d} x\right]^{2}\right. \\
& +v_{1}\left[\int_{X}^{\infty} u_{0}(x) u_{1}(x) \mathrm{d} x\right]^{2} \\
& \left.+2 c_{01} \int_{X}^{\infty} u_{0}^{2}(x) \mathrm{d} x \int_{X}^{\infty} u_{0}(x) u_{1}(x) \mathrm{d} x\right\},
\end{aligned}
$$

where $E_{0}$ is the main-mode electrical field (chosen as real), and where $u_{0}(x)$ and $u_{1}(x)$ are the normalized amplitude profiles of the $\mathrm{TE}_{00}$ and $\mathrm{TE}_{10}$ modes, respectively, given by

$$
u_{0}(x)=(2 / \pi)^{1 / 4} \sqrt{1 / w} \exp \left(-x^{2} / w^{2}\right),
$$

$$
u_{1}(x)=(2 / \pi)^{1 / 4} \sqrt{1 / w} 2(x / w) \exp \left(-x^{2} / w^{2}\right)
$$

where $w$ is the size of the beam. The $v_{0}, v_{1}$, and $c_{01}$ coefficients are given by $v_{0}=\left\langle: \delta P_{0}^{2}:\right\rangle, v_{1}=\left\langle: \delta P_{1}^{2}:\right\rangle$, and $c_{01}=\left\langle\delta P_{0} \delta P_{1}\right\rangle$, where $\delta P_{0,1}$ are quadrature amplitude operators defined in Appendix A. The ":" means that normal ordering is used. The quantity $P_{0}\left(P_{1}\right)$ is associated with mode $\mathrm{TE}_{00}\left(\mathrm{TE}_{10}\right)$. In the following, $V(X)$ is normalized by $E_{0}^{2}$.

The first term of Eq. (1) corresponds to the SQL. The following terms correspond, respectively, to the mainmode noise, the $\mathrm{TE}_{10}$-mode noise, and their correlations. Owing to the normal ordering, the total intensity noise of mode 0 (1) is $1+v_{0}\left(1+v_{1}\right)$. Unity is the SQL, and negative values $\left(-1<v_{0,1}<0\right)$ correspond to sub-shotnoise noise levels. The sign of the correlation coefficient $c_{01}$ depends on the sign convention chosen for $u_{1}(x)$ and on whether the razor blade enters the beam from the left or from the right, since

$$
\int_{X}^{+\infty} u_{0}(x) u_{1}(x) \mathrm{d} x=-\int_{-\infty}^{-X} u_{0}(x) u_{1}(x) \mathrm{d} x .
$$

This point will be discussed further below.

Note that the fits performed in the following of the paper include the noise power $v_{2}$ of the second-order $\mathrm{TE}_{20}$ mode. All the correlations involving this $\mathrm{TE}_{20}$ mode are set to zero. The contribution of this mode is not given in Eq. (1) for the sake of clarity.

The fitting procedure is as follows. The SQL trace is first fitted by an erf function with a horizontal offset and a horizontal scaling as adjustable parameters. The value of these two parameters is then used as a constant in the fitting of the noise trace, where $v_{0}, v_{1}, v_{2}$, and $c_{01}$ are the only four adjustable parameters.

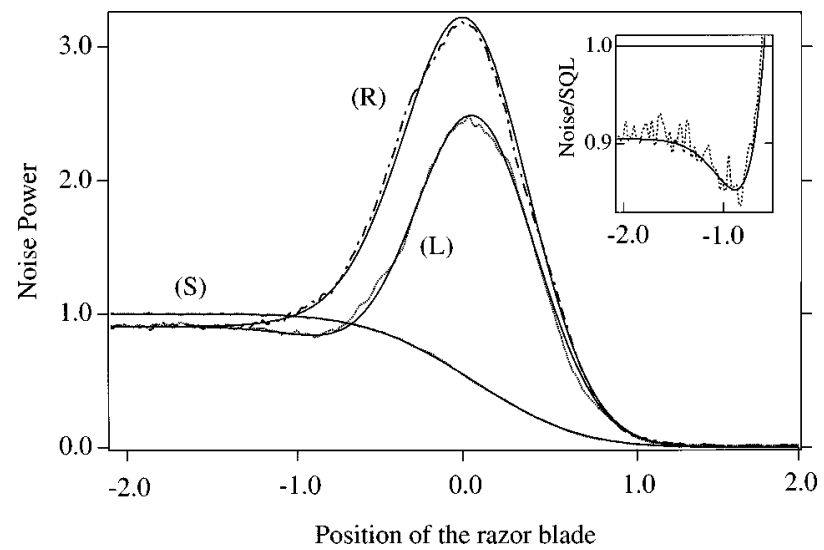

Fig. 3. Razor-blade scan along $x$ (with $w=1$ ). Trace (S) is the SQL (proportional to the intensity). The results of this figure are obtained with a slightly different fine adjustment of the orientation of the grating compared with the results of Fig. 2. Trace (L) [or (R)] corresponds to the razor blade entering the beam from the left (right). For each trace the thin solid curve is the best fit. Trace (R) is flipped over in order to be compared conveniently with trace $(\mathrm{L})$. The fitting parameters are $v_{0}$ $=-0.10 \pm 0.02, v_{1}=14.9 \pm 0.2, v_{2}=1 \pm 0.5$, and $C=0.23$ \pm 0.02 . The inset is a zoom of the SQL-normalized noise of trace (L), showing that the maximum squeezing is obtained when part of the beam is screened. For each trace the thin solid curve is the best fit. 
As can be seen from Figs. 2 and 3, the experimental results are well fitted by this model. The SQL compares favorably with an error function, showing that the mean intensity comes mainly from the lasing $\mathrm{TE}_{00}$ mode. In Fig. 2 the negative value for $v_{0}$ indicates $16 \%$ squeezing. From the value of $v_{1}\left(v_{2}\right)$, it is possible to estimate how far the $\mathrm{TE}_{10}$ mode ( $\mathrm{TE}_{20}$ mode) is from threshold and to infer the excess losses of this mode. ${ }^{15}$ Note that the fits are not very sensitive to the value of $v_{2}$, which explains that the uncertainty on its value is quite large. In Fig. 2 the leftward $(\mathrm{L})$ and rightward $(\mathrm{R})$ scans are nearly identical, in agreement with the nearly vanishing value for the correlation coefficient, given by the fits $C^{L}=0.02$ and $C^{R}=-0.01$, where $C=c_{01} /\left[\left(1+v_{0}\right)\left(1+v_{1}\right)\right]^{1 / 2}$ is the normalized correlation.

The results displayed in Fig. 3 are obtained with a slightly different adjustment of the external grating. The main difference is that the noise profiles are now different, depending on whether the razor blade enters the beam from the left or from the right. The fits are performed in the following way. The arithmetic average of traces (L) and (R) \{i.e., [(L) + (R)]/2\} is first fitted with the correlation coefficient set to zero. One obtains $v_{0}$ $=-0.10, v_{1}=14.9$, and $v_{2}=4$. Each trace is then fitted by holding the values of these parameters in order to get the value of the correlation coefficients. One gets, respectively, $\quad c_{01}^{L}=-0.89\left(C^{L}=-0.23 \pm 0.02\right)$ and $c_{01}^{R}$ $=0.85\left(C^{R}=0.22 \pm 0.02\right)$ for traces $(\mathrm{L})$ and $(\mathrm{R})$, where one should have $C^{L}=-C^{R}$, according to Eq. (4). It can also be observed in Fig. 3 that traces $(L)$ and $(R)$ have different maxima and therefore that the noises on the righthand and left-hand sides of the beam have different values. This behavior is clearly associated with a nonzero value of the correlation coefficient. In the present experiment the physical origin of this asymmetry is attributed to a slight misalignment of the external grating feedback.

In that case an a priori surprising result is that it is possible to improve the full beam squeezing by chopping off part of the beam, as shown in the inset of Fig. 3. More quantitatively, the values deduced from the fit are $v_{0}^{C \neq 0}$ $=-0.10$ and $C=0.22$, while the minimum noise level corresponds to a noise reduction $v_{\min }^{C \neq 0}=-0.15 \pm 0.02$. Moreover, one sees by comparison with Fig. 2 that $v_{\min }^{C \neq 0}$ is almost equal to the full beam squeezing for zero correlation, which is $v_{0}^{C=0}=-0.16 \pm 0.02$. An interpretation of this effect is the following. Owing to the slight grating misalignment, the noisy $\mathrm{TE}_{10}$ mode may leak into the lasing $\mathrm{TE}_{00}$ mode, which creates correlations between them but also increases the noise of the $\mathrm{TE}_{00}$ mode. However, when two quantities are correlated, it is possible to take advantage of these correlations to reduce the noise of one of them; this is just what is done by the partial screening. Quantitatively, the maximum noise-reduction factor that can be obtained from a correlation $C$ is $1-C^{2}$ (see, for example, Ref. 16). This is also in agreement with our observations, since $1+v_{\min }^{C \neq 0} \approx\left(1-C^{2}\right)\left(1+v_{0}^{C \neq 0}\right)$. Correlated excess noise between a lasing mode and a spontaneous-emission mode is clearly reminiscent of the discussions about the origin of the so-called Petermann excess-noise factor ${ }^{17-19}$; this point is developed further in Ref. 20.

All these results are quantitatively confirmed by a sec- ond experiment with a split photodiode (EGG C30822). When the beam is centered on the photodiode, we do indeed observe that the noise difference from the two quadrants is larger than the sum. The difference noise level is directly related to the noise level reached in Figs. 2 and 3 as the razor blade is half way through the beam (i.e., at $X=0$ ). An excess noise in the difference photocurrent is a signature of position fluctuations of the beam. Such an effect has already been observed by Levenson et al. ${ }^{15}$ in a gas laser. In their case, owing to the higher finesse of the laser cavity $(F \approx 200)$, the width of the beat note between the higher-order transverse modes and the main mode is small compared with the free spectral range, and the excess noise in the difference occurs at specific frequencies, whereas the noise spectra can be considered as white in our case.

We also measure directly the correlations between the intensity noises of the $\mathrm{TE}_{00}$ and $\mathrm{TE}_{10}$ modes, which are only inferred from the fits in the razor blade experiment. The experimental setup is depicted in Fig. 4. The laser beam is split into two channels. On channel 1, detector $\mathrm{D}_{1}$ is a split photodiode. When the switch $\mathrm{S}_{1}$ is in position minus (plus), it can be shown that the photocurrent noise power $\left\langle\delta i_{1}{ }^{2}\right\rangle$ is proportional to $1+(2 / \pi) v_{1}$ (1 $+v_{0}$ ). Detectors $\mathrm{D}_{2}$ and $\mathrm{D}_{2}^{\prime}$ on channel 2 make up a usual balanced detection. The switch $\mathrm{S}_{2}$ is used for the shot-noise calibration. ${ }^{13}$ When it is in position plus, the photocurrent $i_{2}$ is proportional to $1+v_{0}$. A signature of a correlation between the $\mathrm{TE}_{00}$ and the $\mathrm{TE}_{10}$ modes would be a change in the noise level on the spectrum analyzer when switching $S_{3}$ from plus to minus, with $S_{1}$ in position minus and $\mathrm{S}_{2}$ in position plus. This is indeed what is observed, in quantitative agreement with the results of the razor-blade experiment.

The same experiment with the scan along the $y$ axis is performed. It appears that in this direction (perpendicular to the junction plane), the noise transverse distribution follows the intensity distribution of the main $\mathrm{TE}_{00}$ mode. There is therefore no contribution from higherorder spatial modes in that direction. It is not surprising that the behaviors along the $x$ and $y$ axes are different since the guiding mechanisms in the diode are clearly different. Here, one can conclude that the guiding is tighter perpendicularly to the junction, but a quantitative explanation of this observation would require a detailed knowledge of the laser manufacturing parameters.

Similar transverse-effects are obtained with this diode in a free-running configuration and with a distributed-

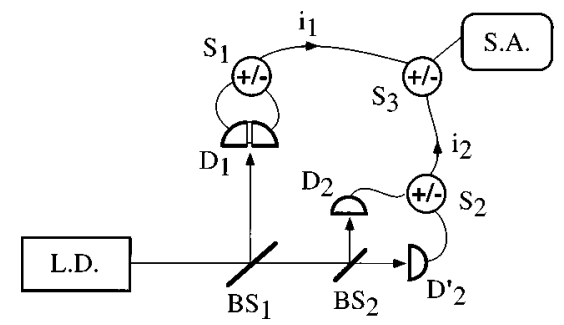

Fig. 4. Correlation experimental setup. $\quad \mathrm{D}_{1}$ is a two-quadrant photodiode. $\mathrm{D}_{2}$ and $\mathrm{D}_{2}^{\prime}$ are ordinary photodiodes. ${ }^{14}$ The transmission of beam splitter $\mathrm{BS}_{1}$ is $70 \% . \mathrm{BS}_{2}$ is the $50 / 50$ beam splitter of the balanced detection. $\mathrm{S}_{1,2,3}$ are RF switchable (+/-) power combiners. L.D., laser diode; S.A., spectrum analyzer. 
feedback laser emitting at $1.3 \mu \mathrm{m}$. We present in detail the result of the external grating configuration because of its sub-Poissonian intensity noise.

We have to emphasize that we rule out several possible loopholes in our experiments. The most severe one is the possibility of having dispersion effects (in the laser or in the optics) converting frequency-dependent behaviors into spatially dependent ones. For this particular gratingstabilized laser setup, some of us have shown that the longitudinal side modes have no contribution to the total intensity noise. ${ }^{9}$ This dismisses therefore any spurious effects of this sort.

As a summary, we have demonstrated the presence of noisy nonlasing higher-order transverse modes in the direction parallel to the plane of a semiconductor laser. This amounts to fluctuations of the beam position along this direction. ${ }^{15}$ It has to be stressed that the effect discussed in this work is different from the anticorrelations observed in Refs. 7 and 9-11, where the involved modes are above threshold. From the two different regimes of Figs. 2 and 3, it appears that transverse-mode control of the laser is a lot more sensitive to external-grating adjustment than the usual frequency control. Finally, the correlations between different transverse modes suggest that the effect we have observed is related, to some extent, to the so-called Petermann excess-noise factor. ${ }^{17-20}$

\section{APPENDIX A: DERIVATION OF EQ. (1)}

In this appendix is presented a derivation of expression (1), giving the intensity noise of a partially screened laser beam along a direction $x$. The laser has a single transverse lasing mode $\left(\mathrm{TE}_{00}\right)$ and several subthreshold higher-order transverse modes $\left(\mathrm{TE}_{i 0}\right)$ labeled $i$.

The electrical field at $x$ is

$$
E(x)=E_{0} u_{0}(x)+\sum_{i=0}^{+\infty} \delta E_{i} u_{i}(x),
$$

where $E_{0}$ is the mean electrical field of the main mode, which is chosen real, and where the $\delta E_{i}$ are noncommuting operators describing the field fluctuations of transverse mode $i$. The field intensity when the razor blade is at $X$ is

$$
I(X)=E_{0}^{2} \int_{X}^{\infty} u_{0}^{2}(x) \mathrm{d} x+\sum_{i=0}^{+\infty} E_{0} \delta P_{i} \int_{X}^{\infty} u_{0}(x) u_{i}(x) \mathrm{d} x,
$$

where $\delta P_{i}=\delta E_{i}+\delta E_{i}^{\dagger}$.

The noise power of $I(X)$ is given by

$$
\begin{aligned}
\left\langle\delta I^{2}(X)\right\rangle= & \sum_{i, j} E_{0}{ }^{2}\left\langle\delta P_{i} \delta P_{j}\right\rangle \\
& \times \int_{X}^{\infty} u_{0}(x) u_{i}(x) \mathrm{d} x \int_{X}^{\infty} u_{0}(x) u_{j}(x) \mathrm{d} x .
\end{aligned}
$$

Defining the function $\Theta_{X}(x)$ as

$$
\begin{array}{ll}
\Theta_{X}(x)=0 & \text { for } x<X, \\
\Theta_{X}(x)=1 & \text { for } x \geqslant X,
\end{array}
$$

the above integrals can be conveniently rewritten as

$$
\begin{aligned}
\left\langle u_{i}\left|\Theta_{X}\right| u_{j}\right\rangle & =\int_{-\infty}^{+\infty} \Theta_{X}(x) u_{i}(x) u_{j}(x) \mathrm{d} x \\
& =\int_{X}^{\infty} u_{i}(x) u_{j}(x) \mathrm{d} x .
\end{aligned}
$$

Finally, with the closure relation $\Sigma_{i}\left|u_{i}\right\rangle\left\langle u_{i}\right|=1$, the noise power can be written as

$$
\begin{aligned}
\left\langle\delta I^{2}(X)\right\rangle= & E_{0}{ }^{2} \sum_{i, j}\left\langle\delta P_{i} \delta P_{j}\right\rangle\left\langle u_{0}\left|\Theta_{X}\right| u_{j}\right\rangle\left\langle u_{i}\left|\Theta_{X}\right| u_{0}\right\rangle \\
= & E_{0}{ }^{2}\left[\left\langle u_{0}\left|\Theta_{X}\right| u_{0}\right\rangle\right. \\
& +\sum_{i=0}^{+\infty}\left(\left\langle\delta P_{i}^{2}\right\rangle-1\right)\left|\left\langle u_{i}\left|\Theta_{X}\right| u_{0}\right\rangle\right|^{2} \\
& \left.+\sum_{i \neq j}\left\langle\delta P_{i} \delta P_{j}\right\rangle\left\langle u_{i}\left|\Theta_{X}\right| u_{0}\right\rangle\left\langle u_{0}\left|\Theta_{X}\right| u_{j}\right\rangle\right]
\end{aligned}
$$

where we use the fact that $\Theta_{X}^{2}=\Theta_{X}$. Defining $v_{i}$ $=\left\langle\delta P_{i}{ }^{2}\right\rangle-1=\left\langle: \delta P_{i}{ }^{2}:\right\rangle$ and $c_{i j}=\left\langle\delta P_{i} \delta P_{j}\right\rangle$, Eq. (1) is then directly obtained from Eq. (A6).

\section{ACKNOWLEDGMENTS}

We thank Christian Bordé for drawing our attention to Ref. 15. This work was supported by the European Strategic Programme for R\&D in Information Technology (ACQUIRE 20029). T.-J. Chang is Boursier du Gouvernement Français.

*E-mail: jean-philippe.poizat@psisun.u-psud.fr.

\section{REFERENCES AND NOTES}

1. S. Machida, Y. Yamamoto, and Y. Itaya, "Observation of amplitude squeezing in a constant-current-driven semiconductor laser," Phys. Rev. Lett. 58, 1000 (1987).

2. W. H. Richardson, S. Machida, and Y. Yamamoto, "Squeezed photon-number noise and sub-Poissonian electrical partition noise in a semiconductor laser," Phys. Rev. Lett. 66, 2867 (1991).

3. M. J. Freeman, H. Wang, D. G. Steel, R. Craig, and D. R. Scifres, "Wavelength-tunable amplitude-squeezed light from a room-temperature quantum-well laser," Opt. Lett. 18, 2141 (1993).

4. Yu. M. Golubev and I. V. Sokolov, Zh. Eksp. Teor. Fiz. 87, 804 (1984) ["Photon antibunching in a coherent light source and suppression of the photorecording noise," Sov. Phys. JETP 60, 234 (1984)]

5. Y. Yamamoto, S. Machida, and O. Nilsson, "Amplitude squeezing in a pump-noise-suppressed laser oscillator," Phys. Rev. A 34, 4025 (1986).

6. A. W. Smith and J. A. Armstrong, "Intensity noise in multimode GaAs laser emission," IBM J. Res. Dev. 10, 225 (1966).

7. S. Inoue, H. Ohzu, S. Machida, and Y. Yamamoto, "Quantum correlation between longitudinal-mode intensities in a multimode squeezed semiconductor laser," Phys. Rev. A 46, 2757 (1992).

8. H. Wang, M. J. Freeman, and D. G. Steel, "Squeezed light from injection-locked quantum well lasers," Phys. Rev. Lett. 71, 3951 (1993). 
9. F. Marin, A. Bramati, E. Giacobino, T.-C. Zhang, J.-Ph Poizat, J.-F. Roch, and P. Grangier, "Squeezing and intermode correlations in laser diodes," Phys. Rev. Lett. 75, 4606 (1995).

10. D. C. Kilper and D. G. Steel, R. Craig, and D. R. Scifres, "Polarization-dependent noise in a photon-number squeezed light generated by quantum-well lasers," Opt. Lett. 21, 1283 (1996).

11. D. C. Kilper, P. A. Roos, J. L. Carlsten, and K. L. Lear, "Squeezed light generated by a microcavity laser," Phys. Rev. A 55, R3323 (1997).

12. C. C. Harb, T. C. Ralph, E. H. Huntington, D. E. McClelland, H.-A. Bachor, and I. Freitag, "Intensity-noise dependence of Nd:YAG lasers on their diode-laser pump source," J. Opt. Soc. Am. B 14, 2936 (1997).

13. H. P. Yuen and V. W. S. Chan, "Noise in homodyne and heterodyne detection," Opt. Lett. 8, 177 (1983).

14. Two photodiodes were used on each arm of the balanced detection in order to avoid saturation.

15. M. D. Levenson, W. H. Richardson, and S. H. Perlmutter, "Stochastic noise in TEM $_{00}$ laser beam position," Opt. Lett. 14, 779 (1989); M. D. Levenson, S. H. Perlmutter, and W. H.
Richardson, "Stochastic position noise, or why a laser beam can not go straight," in Quantum Optics, V, J. D. Harvey and D. F. Walls, eds. (Springer-Verlag, Heidelberg, 1989).

16. M. J. Holland, M. J. Collett, D. F. Walls, and M. D. Levenson, "Nonideal quantum nondemolition measurements," Phys. Rev. A 42, 2995 (1990).

17. K. Petermann, "Calculated spontaneous emission factor for double-heterostructure injection lasers with gain-induced waveguiding,” IEEE J. Quantum Electron. 15, 566 (1979).

18. H. A. Haus and S. Kawakami, "On the excess spontaneous emission factor in gain-guided laser amplifiers," IEEE J. Quantum Electron. 21, 63 (1985).

19. A. E. Siegman, "Excess spontaneous emission in nonHermitian optical system. I. Laser amplifiers," Phys. Rev. A 39, 1253 (1989); "Excess spontaneous emission in non-Hermitian optical system. I. Laser oscillators," Phys. Rev. A 39, 1264 (1989); see also A. E. Siegman, Lasers (University Science, Mill Valley, Calif., 1986).

20. P. Grangier and J.-Ph. Poizat, "A simple quantum picture for the Petermann excess noise factor," Eur. J. Phys. D 1, 97 (1998). 